Voix et Images

voixetimages

\title{
L'ourobouros est un serpent qui se mord la queue X 2
}

\section{Lise Potvin}

Volume 11, numéro 3 (33), printemps 1986

Yolande Villemaire

URI : https://id.erudit.org/iderudit/200577ar

DOI : https://doi.org/10.7202/200577ar

Aller au sommaire du numéro

\section{Éditeur(s)}

Université du Québec à Montréal

\section{ISSN}

0318-9201 (imprimé)

1705-933X (numérique)

Découvrir la revue

\section{Citer cet article}

Potvin, L. (1986). L'ourobouros est un serpent qui se mord la queue X 2. Voix et Images, 11(3), 406-427. https://doi.org/10.7202/200577ar d'utilisation que vous pouvez consulter en ligne.

https://apropos.erudit.org/fr/usagers/politique-dutilisation/ 


\title{
L'ourobouros est un serpent qui se mord la queue X 2
}

\author{
par Lise Potvin, Université du Québec à Rimouski
}

L'ouvre nouvelle ne diffère de l'ouvre ancienne que par l'écume d'époque. Le fond est le même: la vie qui vit vive...

P. Haeck

Tout cristal nous guette. Si entre les quatre Murs d'une chambre, se trouve un miroir, Je ne suis plus seul. Un autre est là, le reflet Que dispose dans l'aube un théâtre secret.

\section{J.L. Borgès}

La modernité est avant tout un acte de lecture.

N. Brossard

Comment parler de ce roman rotatif et encyclopédique sans l'arrêter dans sa course giratoire? Comment décrire la Vie en prose en laissant intactes et la vie et la prose qui bougent au rythme échevelé de la modernité? Placer sur la lame du microscope la chose agitée pour en étudier les contours et les couleurs équivaut, d'une certaine manière, à réduire son champ d'investigation. Sachons-le. Renonçons dès le départ à tout voir et à tout saisir. Sans laisser entendre pour autant que de ce livre nous ne pouvons rien dire.

D'abord, que la prose prenne la pose! Flash sur le titre que l'on s'amusera à découper; éclairage discret sur la position de qui manie la lampe et la loupe. Mais peut-être faudra-t-il quitter ce poste d'observation, somme toute confortable, pour répondre à l'invite du texte qui fait signe et qui entraîne. Difficile de résister. Tire la chevillette, la bobinette cherra ${ }^{\prime}$, conseille Minerve après avoir stoppé la machine (wow les moteurs). Le lecteur a beau prendre garde à la fiction, le voilà qui déboul[ $e$ ]dans l'histoire comme Gombie dans le livre d'images (p. 55). Comment (s'en) (sans) sortir? À la recherche d'une issue, trois fois passera... à travers le texte, et sans doute plus souvent encore. (Pourquoi pas huit?) Il errera par plus d'un chemin, confondant les pierres précieuses et celles qui brillent d'un éclat trompeur, ébloui par tous les tons de la palette mais sachant bien que l'important c'est le/la rose suggéré(e) par la prose. Et le carrousel de tourner longtemps avant que n'apparaisse l'exit, stroboscopique et basculé de biais. 


\section{Le titre, à titre illustratif}

Le texte, écrit Jean Ricardou, est $[\ldots]$ une machine à lire son titre ${ }^{2}$. Dans le même ordre d'idées, il précise: Si le titre tend à unifier le texte, le texte doit tendre à diversifier le titre, à le faire exploser en le soumettant à une multitude de définitions ${ }^{3}$. Au jeu du découpage, la Vie en prose fait voir sa polysémie.

Supprimant le " $\mathrm{p}$ », on obtient: la vie en rose. Le roman annonce sa (ses) couleur(s). Le rose prédomine. Rimbaud et Aquin donnent le ton dans ce roman où le rose coule en larmes au milieu du papier blanc (ça dérape sur un papyrus rosé) et où la nourriture s'harmonise au décor (on mange des choses roses). Mais une couleur en appelle une autre jusqu'à épuisement du paradigme. De la plus sage à la plus folle (black-watch, tango), elles irisent ou bariolent le texte. Rose cite le «Sonnet des voyelles» mais nous informe qu'elle est plutôt à la recherche de la couleur des consonnes.

"La vie en rose", c'est aussi la chanson d'Édith Piaf, côté cœur un peu naïve, un peu fleur bleue côté jardin. Comme le roman en quelques-unes de ses facettes. Le disque tourne à plusieurs reprises. Le roman se fait boîte à musique. Une kyrielle de chansons, chansonnettes, comptines agiront comme embrayeurs du récit ou comme moteurs activant le manège sur le point de s'arrêter. Au bout de cinq ou six semaines, les phrases vinrent vinrent vinrent à manquer. On tira à la courte paille pour savoir qui qui qui... relancerait l'action ( p. 68). Les ritournelles enfantines et les succès du palmarès voisinent avec les formes littéraires les plus sophistiquées. Belle sarabande baroque.

Le rose renvoie au féminin. On pense au magazine féministe ${ }^{4}$ : le roman en a la couleur et la joyeuse philosophie. Livre de femme(s) qui ne théorise ni ne prêche mais qui met en jeu une façon d'être qui est une façon de vivre qui est une façon d'écrire. Pas de discours revanchard ou victimisant: les femmes de la vie et de la prose s'organisent, se serrent les coudes, apprennent à se défendre (Wen-Do). Femmes et fortes? Ben oui.

La vie en rose, c'est également la vie vue par Rose alias Yvelle Swannson: un personnage sporadique, un space-opera phosphorescent.

Le titre, avec son «p» retrouvé revient à la prose. Les protagonistes se bousculent pour nous donner à lire leurs phrases et leurs gloses, leurs lettres, leurs manuscrits avec, en prime, leur tourment et/ou leur jubilation devant la page vide. Prose aux prises avec elle-même, prose en proie à sa propre analyse.

La Vie en prose convoite aussi la prose des «autres». Un vaste réseau de renseignements intertextuels assure une mainmise sur la Bibliothèque. Non déclarés, une série d'emprunts, de citation, de références, circulent impunément dans le texte. Todorov, Dante, Erica Jong, Mallarmé, Laure Conan, Agatha Christie et autres préférés composent une "Bibliothèque de Babel» dont Borgès aimerait l'agencement. 
Selon le doublet traditionnel qui l'oppose à la poésie, la prose connote le réaliste, le banal, le terre à terre. La Vie en prose joue sur ce versant des choses mais privilégie l'écriture, le "côté hiéroglyphe» du réel qui permet à tout moment aux personnages du quotidien de basculer dans l'envers du décor, là où le roman ouvre sur le merveilleux, l'orient, l'hyperespace et autres contrées promises.

R rose Sélavy se cache dans le titre et dans le texte avec tous ses mystères. Elle annonce et met en branle les mécanismes perturbateurs de la pensée logique 5 . Ludique, masquée, magique comme chez Desnos, elle est le plaisir du texte, la folie rose, la "tour de babil" du langage: consonnes qui cliquètent, sons «lâchés lousses», mots livrés à eux-mêmes pour voir de quoi ils sont capables. Mécanique cacophonique ou bruissement harmonieux. C'est selon.

La Vie en prose c'est "la vie qui vit vive» mais c'est aussi l'exploration romanesque de la différence (l'écart, l'opposition) entre la vie et la prose, les mots et les choses, le réel et la fiction. On veut apprendre à distinguer entre les mots et les choses, à cesser d'avoir peur des uns quand [on a] peur des autres... (p. 157); pourtant l'on sent que du réel jamais les mots ne sauront rendre compte (p. 120).

Au lieu de la rupture, c'est parfois la (con)fusion entre vie et prose qui est considérée, le joint entre réel et fiction, le lien entre mot et chose. La distinction n'existe pas (p. 48 et p. 142). Il arrive qu'on "doute du réel», ce qui revient [...] au même qu'à douter de la fiction (p. 120). Au jeu de la dissemblance/ressemblance, le titre fait écho. Il s'agit de dire la vie en prose, les choses en mots, le réel en fiction: la préposition sert de pont entre les deux rives, marquant à la fois leur rapport et leur hétérogénéité. On dirait qu'il y a des ponts entre la fiction et le réel... (p. 150), mais ils sont toujours à inventer, à reconstruire, à défendre (p. 174). Entre vie prose, choses mots, réel

fiction, il y a cet «en» ("entre», "antre $»$ ), lieu énigmatique du passage à l'écriture.

Titre aux plis nombreux, titre dense, dru, serré. En même temps que dépouillé, abstrait: il pourrait coiffer toute entreprise romanesque. Une telle polyvalence donne le vertige. C'est bon signe. Villemaire confie: Mon écriture n'a de sens que là où elle vous branche sur un spin et vous ouvre le réseaú.

\section{Précis d'optique}

Raymond Montpetit, esthéticien, sémiologue, propose d'aborder un texte à partir de l'interrogation suivante: comment fonctionne la machination particulière qu'est cette écriture ${ }^{7}$ dont je veux rendre compte? Vouloir répondre à cette question, c'est entrer soi-même dans la machination que l'on lit, en l'écrivant et en la remettant en jeu ${ }^{8}$. Il s'agit d'une collation de deux machines d'écriture: la mienne - l'autre et d'une réactivation du texte initial; gestion textuelle qui oblige à une constante proximité avec l'œuvre étudiée. 
Si l'on dit que le sens découle d'un certain jeu (non seulement dans le sens ludique du terme mais de même que les différentes pièces d'un mécanisme jouent les unes sur les autres), il devient possible, «à l'occasion d'un texte", de démontrer quelques-uns des rouages, de montrer leur engrenage et comment le sens est produit dans ces jointures comme un effet de la manière dont tout cela est mis en branle et fonctionne ${ }^{9}$. Le texte fouillé dans ses articulations apparait comme une machine savamment montée pour produire ses effets ${ }^{10}$. Effets de réel, de sens, de forme, etc. Tout ce que le livre donne à lire est pris en considération. La machination critique refuse de choisir entre l'analyse du signifiant et la quête du sens, elle cherche à entrer dans le jeu machinal du texte qui met en scène et fait fonctionner à la fois les thèmes, les personnages, les structures et les sens, autant la fiction que la narration...". Elle est la mise en écriture du fonctionnement des composantes de l'œuvre.

Les textes de Villemaire appellent une telle gérance critique. A la question "pourquoi écrire aujourd'hui?" elle répond: pour interroger la machine du langage ${ }^{12}$. Elle ajoute: pour le plaisir de taire certaines choses. Délice de la dissimulation qui n'est pas sans rappeler Derrida pour qui $[u] n$ texte n'est un texte que s'il cache au premier regard, au premier venu, la loi de sa composition et la règle de son jeu ${ }^{13}$. À tenter d'ordonner les pièces du puzzle, plusieurs lectures sont possibles. Mais jamais définitives. L'œuvre est un processus dynamique qui réserv[ erait] toujours une surprise à l'anatomie ou à la physiologie d'une critique qui croirait en maîtriser le jeu, en surveiller à la fois tous les fils... ${ }^{14}$. Sagesse critique qui invite à focaliser sur quelques éléments de la trame textuelle.

Le texte, faut-il encore ajouter, s'apparente à l'activité onirique. Le travail du texte est semblable à celui du rêve, suggère Montpetit. L'un comme l'autre ne jugent pas; ils se contentent de transformer: condensation, déplacement, distorsion, déformation. Le travail du rêve, le travail du texte orchestrent un jeu de transformations, de substitutions.

Entrer dans le jeu numéroté (les pages, mais dans quel ordre?) de la Vie en prose ce sera remonter la série substitutive, à la recherche du premier terme qui toujours se dérobe dans un tourbillon de figures, dans une sorte de rallye textuel où l'erreur est possible à chaque virage. Nous sommes prévenus. Let's go.

\section{Entrez, entrez dans la danse...}

Un premier parcours de la Vie en prose déjoue les attentes, brouille les cartes. Une lecture linéaire du "trajet enchevêtré de ce signe de piste" rencontre des obstacles, bute sur des contradictions, spatiales et temporelles, entre autres. Une foule de personnages dont on ne parvient pas à reconstituer l'identité s'interpellent à travers deux continents, en des temps qui ne coïncident ni ne s’ajustent. Un jeu complexe de relais en manière de farandole pour une écriture sans fin. 
Reprenons du début. Lecture minutieuse, attentive aux indices. La machine substitutive est déjà en action. Le premier chapitre du roman "Ton nom de Los Angeles dans mon réel désert" (qui évoque Ton nom de Venise dans Calcutta désert de Marguerite Duras) est précédé d'un incipit. Scène présentée par une narration neutre qui donne la parole à une douzaine de personnages, des femmes, réunies, pour en juger, autour du manuscrit de Noémie Artaud. Ce qui frappe: l'instance narrative. Vision du dehors qui se contente d'enregistrer (au moyen du style direct, indirect ou indirect libre) le discours des protagonistes; ce qui aura pour résultat de nous renvoyer à l'excipit, conçu sur le même modèle.

On remarque que l'ouverture du texte (Vava dit que, bien sûr, il y a des choses qui arrivent, et tout $c^{a}$ ) en rejoint la fermeture. La première phrase du roman recoupe l'avant-dernière. Un cercle. A y regarder de près, on voit que cette répétition joue en même temps au niveau d'un autre texte: le manuscrit de Rose, Yvelle Swannson, dont on s'apprête à corriger les épreuves. Deux romans aux points d'arrivée et de départ confondus. Une double boucle. La figure du huit vient à l'esprit, confirmée par des interventions insinuantes:

Solange regarde le manuscrit de Rose avec l'octascope que Laure lui a rapporté de San Francisco. Dit [...] que ça multiplie le réel cette lunette-là. Ensuite Carla regarde Solange dans l'octascope, dit qu'il y a huit Solange qui lui sourient à la fois. Huit fois la même amour...

Elles sont nombreuses, disséminées. Laure chante sur tous les tons de tous les octaves, dessine des huit à l'infini et veut écrire à sa sœur sans y mettre huit ans (p. 69 et p. 67). Plus loin, on lit:
Mon frère aura, samedi dans cinq jours, vingt-six ans [... Je dis ça parce qu'en calcul théosophique, ça donne huit [...] Le huit, ce n'est pas que l'infini, c'est aussi la découverte des moyens et la matérialisation ${ }^{15}$.

Voilà qui encourage à continuer dans cette voie. La représentation du chiffre huit superpose deux cercles, rond dédoublé, multiplié ou encore réfléchi par un miroir. Or le roman trace et anime un nombre incalculable de figures circulaịres, sphériques, rotatoires tandis qu'il se couvre de surfaces réfléchissantes.

Le chapitre quatre s'intitule "On y danse tout en rond". Les personnages s'amusent à faire des ronds dans l'eau et des ronds de fumée pour oublier qu'ils sont parvenus au centre de rien, même si de là, [on] voi[ $t]$ bien la circonférence (p. 175). Le principe de la circularité s'étend aux références culturelles (on cite le Cercle de craie caucasien de Brecht) et à l'espace individuel ( $j$ 'envahissais dangereusement sa sphère vitale $\mathrm{p}$. 197). Pendant que tourne $[\ldots]$ cette roue qui nous enferme dans la matière (p. 141), Rose, dans son space-opera, fait défiler les planètes alors que Lexa et sa compagne s'évadent en altitude dans le restaurant tournant quifait deux tours (p. 149). 
Par son redoublement syllabique, le premier mot du texte (Vava) qui est en même temps le dernier, place le roman, d'entrée de jeu, sous le signe de la répétition. Something so strange... Right there in the mirror (p. 18). Le dispositif est en place, la série des effets optiques peut s'enchaîner: reflets, dédoublements, substitutions, prolifération. Sabada Dabasa inverse l'écho de son nom; le chat de John s'appelle à lui seul Istamboul et Constantinople; les jumeaux, les jumelles se confondent; la liste des sosies s'allonge. Passe Aurélia suivie-des-filles-du-feu.

Alice fait du miroir ce qu'elle veut (p. 99). Il opère à plus d'un niveau; c'est lui qui commande une sorte de bipartition du roman. L' ouvre renvoie constamment à "un envers du décor", "un autre temps du verbe», "un autre côté du miroir ", suggérant deux univers coextensifs. L'une des protagonistes admet: C'était beaucoup plus magique quand j'ignorais encore le versant noir qu'on ne voit que lorsqu'on a traversé la frontière (p. 142); l'autre comprend que le secret des Changeurs de signes est impossible à déchiffrer de ce côté-ci du miroir (p. 183). Où donc est la ligne de partage?

$\AA$ une première approche systématique, se dégagent quelques constantes: le huit, le cercle, le miroir. Il a fallu choisir. On aurait pu retenir le triangle, la pyramide, le losange. On aura compris que dans ce roman sémiologique, c'est la surabondance de signes qui fait problème. Sous l'avalanche, nous avons opté pour les configurations les plus évidentes.

Mais l'œuvre est loin d'avoir livré toute sa complexité formelle. Nous en sommes toujours à examiner les rouages élémentaires de la machine fictive, constamment déportés, distraits, entraînés sur de nouvelles pistes au cœur d'un récit tentaculaire et sibyllin.

Récapitulons. Une même optique narrative ouvre et ferme le roman. Cette vision neutre d'un témoin non identifié, non compromis dans l'action se retrouve à un seul autre moment du texte ${ }^{16}$, formant trois régions narratives qui, par leur facture, se détachent de l'ensemble du roman. Grosso modo, ces trois séquences permettent d'assister à trois rencontres d'un groupe d'amies, de femmes qui pratiquent, pour la plupart, entre autres métiers, celui d'écrire, rencontres qui ont lieu autour de textes à lire, à corriger, éventuellement à publier. Conscience narrative unifiante, adéquation des personnages avec eux-mêmes (fidélité d'une séquence à l'autre de leurs traits de personnalité, de leurs tics de langage), identités données en clair, voilà les caractéristiques de ces trois scènes qui constituent une sorte de fiction au premier degré par rapport au reste du roman qui, sans avertissement, nous propulse à travers des manuscrits multiples, aux contours mal définis, où les auteurs gardent l'incognito, où les personnages, réfractés, déguisés, jouent à cache-cache avec leur propre image. Il nous faut des points de repère pour circuler dans le labyrinthe; ils viendront, pensons-nous, des renseignements tirés de ces passages exempts de contradictions.

Que nous apprennent-ils, concrètement, sur les personnages? En résumé, ceci. Solange est en train d'écrire la Vie en prose, Rose est l'auteure d'Yvelle 
Swannson. Quelques identités se précisent: le véritable nom de Nane c'est Danielle Yelle; sa sœur Noëlle s'appelle en réalité Noé-Vladimira Yelle (un anagramme de Yolande Villemaire). Vava se nomme Eva-Vera Indianapolis; pseudonyme: Noémie Artaud. Nane signe le Livre-Sphynx du nom de Gloria Olivetti. Lotte (Charlotte) a des jumelles Bip et Pola (Bibiane et Pauline) ainsi nommées d'après Bouvard et Pécuchet. Elle est la sœur de Laure (de son nom d'Aurel, Lisle, Aurélia). La question de l'identité qui travaille le roman de part en part montre ici un peu de sa complexité. Les trois séquences renseignent en outre sur l'espace. Notons les déplacements: Laure a sẻjourné en France; Rose est allée à Amsterdam; Lotte et Laure connaissent NewYork; Solange, Nane et Laure ont vu la Californie. Rose a un chat qui se nomme Leïla. Autant d'indices qui nous permettront d'identifier (are you sure?) les personnages doublement fictifs qui se baladent dans les manuscrits et les auteures qui se dissimulent derrière les pronoms.

Reprenons la lecture, dorénavant avertie des écueils, nantie de fiches signalétiques.

Le premier chapitre se divise en douze scènes. Les cinq premières se passent en Californie. Une jeune femme dont l'identité ne nous est pas révélée (mais nous savons maintenant que Solange, Nane et Laure ont déjà voyagé en Califonie) tente d'y oublier une histoire de cœur. Désœuvrée, elle erre de bar en bar. Elle rencontre un certain Bryan, l'ancien chum d'une amie de sa sceur. (S'agit-il de Nane ou de Laure qui ont chacune une sœur?)

La scène six rompt la continuité. Une narratrice en train d'écrire l'históire de Nane Yelle (c'est parti, il fallait un début [...] ça a bien failli commencer par: Nane Yelle est née ici, à $M$. Réal) nous fait part de ses humeurs, de sa technique romanesque, de ses lectures, des manies de son chat. Qui est-elle? Solange? Rose? ( $\grave{A}$ cause du chat? Mais l'animal n'est pas nommé et nous savons qu'au moins trois autres chats se sont glissés dans le roman - Mootje, Eurydice, Istamboul et Constantinople - qui témoignent de l'identité de leurs propriétaires).

La scène sept se présente comme une page de journal avec en-tête:«Los Angeles, 28 juillet 1976». Notre héroïne du début cherche consolation à Hollywood. Elle écrit: C'est chaud et humide. Plus loin dans le roman, nous tiquerons sur le passage suivant daté du 10 août 1978: Ce matin, j'ai reçu une carte postale de Solange qui voyage en Californie cet été [...] Solange me dit que la sécheresse est terrible en Californie (p. 91). Problème.

À la huitième scène, on apprend que notre touriste tient un journal de bord. A Disneyland, elle prend un billet pour Adventure Thru Inner Space. Étrange circuit, que l'on a envie de comparer à celui que nous décrivons dans l'espace romanesque:

Il n'y a pas le moindre point de repère. C'est noir, noir, noir, et ça dure longtemps. Et puis, lentement, des points lumineux apparaissent... 
La scène neuf, courte et ambiguë, laisse dans l'indécidable. La présence de Leïla, voluptueusement étalée sur des feuilles de papier permet d'attribuer ce passage à Rose mais avons-nous affaire à la romancière du début (qui écrit l'histoire de Nane Yelle) ou s'agit-il d'une nouvelle immixtion dans la trame du récit?

Les scènes dix et onze ramènent en DC 8, de ' $A$ à Montréal, l'héroïne inconsolée.

L'écrivaine de la scène six rédige la dernière séquence: on reconnaît son style, ses préoccupations. Pas de nouveaux indices. Nous n'arrivons toujours pas à établir clairement les identités. Qui voyage en Californie? Qui raconte? Nane? Rose? Solange? Dans le titre du chapitre, il y a "Los Angeles", dans "Los Angeles" il y a "Solange", il y a même "les Solange". Seraient-elles plusieurs à écrire des histoires qui se ressemblent?

La lecture linéaire, on le voit, n'est pas très éclairante. Aux problèmes de narration s'ajoutent, dans les chapitres subséquents, des superpositions d'espace, des intrications temporelles. Depuis le début, d'ailleurs, le temps fait des siennes. Il lui arrive de drôles de choses (p. 42). Dans l'incipit, les personnages discutent du film Pink Lady, pourtant ce n'est qu'au chapitre onze qu'aura lieu la première. Souvent obsédants (pour nous lancer sur des fausses pistes), les supports temporels se font parfois très discrets (on situe le dernier chapitre à l'été 79 parce que les personnages craignent les retombées du Skylab). Il arrive que les horloges s'arrêtent, comme par un effet de bonsoir à retardement comme une bombe posée sur la track du temps qui fait des boucles comme le ruban d'un magnétophone jammé... (p. 175). Personne ne s'inquiète (sauf le lecteur) car il est admis qu'à cette altitude, le temps est rond (p. 201).

Il y a un bon moment déjà que le lecteur joue avec l'idée de lire ce roman dans un autre ordre que celui de la succession paginée. Mais lequel? En y dessinant des huit? des cercles? En ce cas, par où et comment commencer? Dans l'incertitude, pourquoi ne pas interroger le sphynx...

\section{Tire la chevillette et la bobinette cherra}

Le Livre-Sphynx, au centre de l'œuvre, brise la structure romanesque rencontrée jusqu'ici. Au morcellement spatial, temporel et narratif des autres chapitres, il oppose une unité de lieu, de temps, d'action. Ȧ travers l'apparent désordre des flash-backs et des analepses, on repère un lieu unique (l'Italie), une chronologie sans faille (l'été 78), la poursuite d'une même activité (l'assistance à un colloque qui s'étale sur quelques jours) vécue et racontée par une seule narratrice.

Elle n'a pas de nom, bien entendu. Nous l'appellerons $\mathrm{Y}$. La lettre $\mathrm{X}$ étant déjà mobilisée, dans le texte qu'elle nous donne à lire, pour désigner son compagnon. Son frère dans le roman s'appelle $\mathrm{Y}$ mais nous n'hésitons pas à surdéterminer la lettre puisqu'elle écrit: 
[ Les initiales de] ce nom qui est le mien [...] sont identiques à celles de celui de mes frères qui m'a dit qu'il racontait à ses copains de Vancouver que c'était lui qui avait écrit ce premier roman que j'ai publié et sur lequel l'éditeur, à mon grand désarroi, n'avait inscrit que l'initiale de mon prénom.

Lieu du commencement, le livre-Sphynx «semble» l'être qui débute par une sorte de degré zéro de la fiction où l'écriture attentive à son propre tracé, discourt sur la lettre «a», l'aleph, le «a noir» de Rimbaud, l'archétype (de la Grande Mère), principe originel (p. 120).

Peu à peu, il «livre» des réponses. Mais des réponses en forme d'énigmes. Il tend des clefs, ne dit pas quelles sont les bonnes.

Certaines pistes sont plus évidentes. On apprend qu'Y. profite de son séjour en Italie pour mettre de l'ordre dans ses textes. Renseignement capital: elle dactylographie la Vie en prose en même temps qu'elle travaîlle au manuscrit du Livre-Sphynx. Ironiquement, elle explique:

Mes futés lecteurs ne manquent sans doute pas de remarquer certaines intertextualités entre ce texte-ci auquel je donnerai le titre de Livre-Sphynx [...] et certains chapitres de la Vie en prose $[. .$.$] que vous aurez lus au moment où vous lirez ceci...$

(p. 150)

Voilà qui jette un peu d'éclairage sur les contrepoints narratifs disséminés dans le roman. On constate également qu'Y. a beaucoup voyagé: la Californie, New-York, la France, l'Angleterre, le Maroc, etc. Bref, défilent dans ses réminiscences tous les lieux parcourus en d'autres chapitres par des personnages distincts.

Parmi la longue liste de ses souvenirs, retenons le plus curieux. Sur une plage anglaise, elle aperçoit une pierre qui de loin ressemble à un sphynx. De près, la pierre a du relief d'un côté, de l'autre elle a la forme d'un livre ouvert. Influencée par la pensée magique qui imprègne les héros de Castaneda (qu'elle est en train de lire), Y. va écrire son nom sur la pierre avec une solennité qui confère à la scène, et à la signature, un caractère rituel.

Certaines autres pistes donnent plus de fil à retordre et ne se comprennent qu'à partir du moment où le lecteur, dans sa recherche du fil d'Ariane, décide de disposer le roman en cercle, comme une horloge dont les heures seraient marquées par les numéros de chapitre. (Comme le roman contient onze chapitres, l'incipit reçoit la marque zéro - puisqu'il précède le premier chapitre - et il prend la place du chiffre manquant.)

Dans cette optique, une foule de consignes de lecture, jusqu'ici lues au premier degré, se font jour et éclairent la structure du roman. Le LivreSphynx (chap. 7) aligne plusieurs prescriptions textuelles. Une phrase 
comme: Lève les yeux, lecteur et fixe-les au point / Où sont entrecroisés deux mouvements contraires ( p. 164), empruntée à Dante, possède une fonction dans l'économie du récit commande, à un autre niveau, la marche de la lecture. Deux manuscrits s'imbriquent et vont dans des sens différents. C'est tout ce que la consigne nous apprend. Mais on déduit que le sens des flèches est forcément celui indiqué par le tableau car emprunter

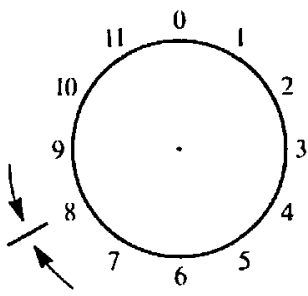
la direction contraire mène à une impasse formelle: le chapitre 7 (contrairement au chapitre 3 , par exemple) ne peut se lire en commençant par la fin (on assisterait à la clôture du colloque, en allant peu à peu vers son ouverture).

Autre indication d'importance: la narratrice insiste, en trois endroits différents, sur l'heure que marque la montre Timex dans la réclame télévisée, (deux heures moins dix). Après force commentaires, elle précise qu'elle en voit le sens qui consist $[e]$ à décoller la grande aiguille pour qu'elle traverse l'espace qu'il y a entre le dix du cadran et le deux qu'on nomme dix quand il est désigné par elle...

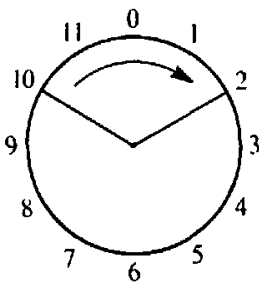

(p. 124). Pour s'assurer que le lecteur n'a pas laissé passer cet élément essentiel, elle revient à la charge:

[...] le dispositif dix heures dix ou deux heures moins dix a au moins l'avantage d'ouvrir vers le haut comme dans ce geste des bras que font les charismatiques [...] comme dans le V du peace des flower children, comme dans la figure que forment les deux bâtons que tient dans sa main, la jeune femme de l'arcane XXI du Tarot de Marseille, celle qui marche dans un cercle comme un cobaye dans sa roue.

Lire cette partie du roman (chap. $10,11,0,1)$ en suivant la consigne s'avère rentable. On comprend maintenant pourquoi les personnages discutent de Pink Lady à l'incipit (chap. zéro): ils viennent d'assister à la première (chap. onze). 
La narratrice utilise le même code lorsqu'elle nous dit avoir envoyé à pré-

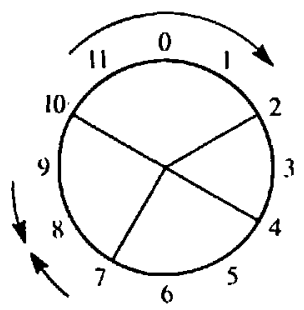

cisément sept heures vingt, parce que ça fait partie du jeu ${ }^{17}$, une carte postale à son jumeau. Nous reproduisons sur un même tableau les instructions recueillies jusqu'ici. Elles opèrent une première division du roman qui laisse cependant plusieurs points dans l'obscurité. La nouvelle ligne de démarcation qui vise le chiffre quatre

nous incite à relire ce chapitre intitulé (ce n'est pas au hasard) «On y danse tout en rond". Il contient des renseignements précieux:

...le roman, comme le cahier, devrait commencer sous le soleil exactement, dans le bleu bleu bleu du ciel de Provence, et s'enfuir dans la lavande quand le temps se fait nuageux avec périodes ensoleillées ou ensoleillé avec périodes nuageuses ${ }^{18}$.

Deux pages plus loin, un nouveau chapitre ( «Précis d'énergie solaire») dont l'action se déroule en Provence, s'ouvre sous le soleil exactement. Ce sera notre point de départ. Et nous lirons en marche arrière puisque la consigne suggère de filer dans la lavande (l'avant de). D'ailleurs, le bulletin météorologique qu'elle contient n'est pas gratuit; on le retrouve entier, deux paragraphes plus haut, sur la même page (nouvel indice qu'il faille aller dans ce sens - le temps s'y faisant nuageux avec périodes ensoleillées ou ensoleillé avec périodes nuageuses) comme réfléchi par le truchement d'un miroir. Nouvelle machination de reflets, narration par miroir qui, au premier abord,

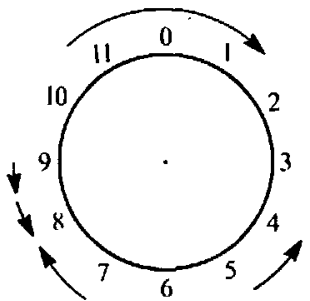
déroute le voyageur engagé dans cette "Adventure Thru Inner Space». Sa fonction s'éclaire dans l'optique du rallye textuel: il s'agit d'un panneau qui commande de rebrousser chemin. Au milieu du chapitre 9, une autre consigne suggère la rétrogradation: $j e$ tire des lignes sur le papier blanc de ma vie qui se débobine par en ar-

rière... (p. 201), écrit la narratrice. Le tableau ci-dessus reproduit toutes les flèches de direction montrant bien que certaines parties du roman sont laissées sans signalisation routière. Les chapitres 2 et 3 , par exemple, en raison d'une ambivalence chronologique et structurelle remarquable, se lisent dans les deux sens. Selon le chemin qu'il emprunte (les diverses consignes de lecture autorisent plus d'un trajet), le lecteur aura à les traverser dans un sens ou dans l'autre, investissant différemment les signes rencontrés (ils sont délibérément flous) s'il vient de la droite ou de la gauche.

Avoir reconstitué la marche turbulente de la lecture ne règle pas tous les problèmes. Combien y a-t-il de manuscrits? Où sont leurs frontières? Qui écrit? Une batterie d'indices permet de conclure que le roman se divise en 
deux livres principaux (la Vie en prose et le Livre-Sphynx - les deux côtés du miroir, l'avers et l'envers du décor, l'une et "l'autre galaxie»), lesquels contiennent chacun en plus de leur propre substance, deux autres manuscrits (celui de Noémie Artaud, les écrits de Noëlle, Yvelle Swannson et «l'écriture rouge»). Au total, six textes, six auteures. Devant la complexité du produit, Y. avoue: Pour moi le deux binaire du duel et le six démoniaque et pluriel $[\ldots]$ ont été $[\ldots]$ des catapultes danis des techniques d'enchâssement dont je ne suis pas certaine de sortir réelle (p. 219).

Sur tableaux circulaires, on obtient les arrangements suivants:

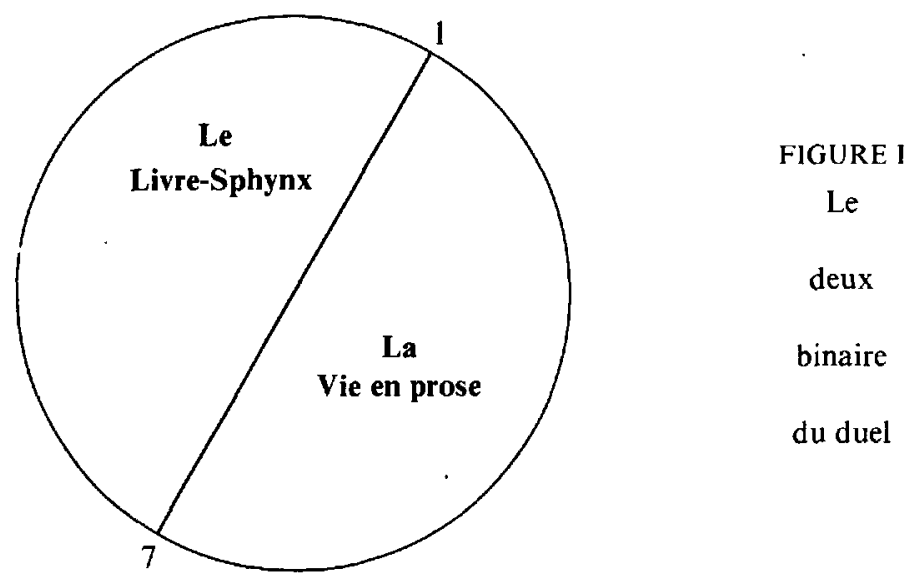

$$
\begin{aligned}
& \text { FIGURE II } \\
& \text { Le six } \\
& \text { démoniaque } \\
& \text { et pluriel }
\end{aligned}
$$

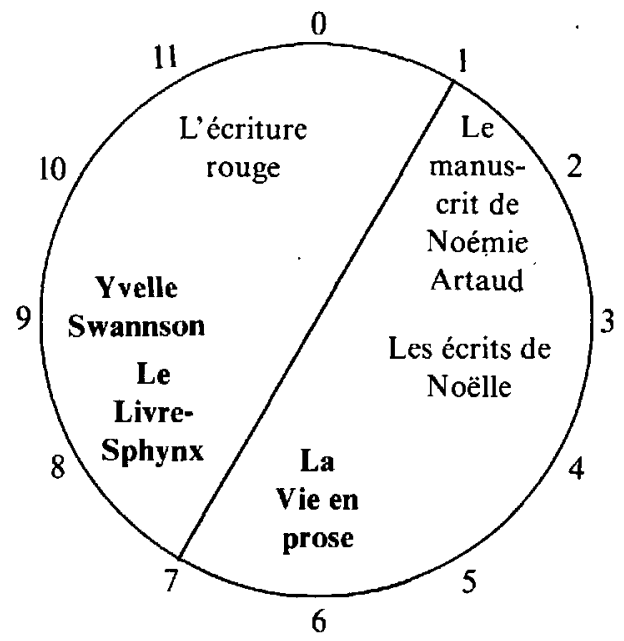


C'est délibérément que la figure II omet de marquer la frontière entre les différents manuscrits. Elle varie selon l'interprétation du lecteur, selon les consignes qu'il privilégie. Ainsi, le manuscrit de Noémie Artaud occupe le premier chapitre du roman si l'on considère qu'il ne contient que les aventures de Los angeles ${ }^{19}$. Or, rien n'empêche de penser, vu la réversibilité des signes et l'ambiguitté des messages ${ }^{20}$, qu'il s'étend sur les deux premiers chapitres. Bref, la structure est mobile, malléable. Une organisation différente des mêmes données conduit le lecteur à des résultats différents.

La lecture proposée ici serpente à travers les six manuscrits, guidée à chaque détour par des poteaux indicateurs, encouragée au passage par la conviction d'un personnage (cela s'enchaine elle en est certaine p. 80) ou par le miroir qu'un autre lui tend:

Je m'installe pour lire. Au bout de deux pages, je tombe sur: "Une fois le dernier mot écrit, le roman donnerait l'impression de se tenir dans tous ses morceaux, on y devinerait une intention précise, une chronologie et un temps particuliers, comme si le livre ne pouvait pas être différent de ce que je l'avais fait devenir».

(p. 220)

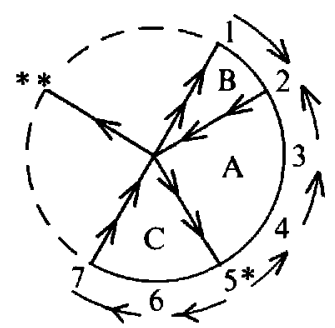

\section{D'abord la Vie en prose}

Elle commence sous le soleil exactement $\left(^{*}\right)$ par les écrits de Noëlle (A) avant de passer par le manuscrit de Noémie Artaud (B) et de redescendre en $5,6,7$ pour que son dernier chapitre (C) s'écrive les yeux dans les yeux avec le Sphynx (p. 257). Le dénouement, étalé sur quelques pages, aura lieu dans le Livre-Sphynx $\left({ }^{* *}\right)$,

rappelant les représentations du Tao dans lesquelles on retrouve une partie du Yin dans le Yang et vice versa ${ }^{21}$. Un ensemble de signes convergent vers la fin du chapitre 9 pour la désigner comme scène finale du récit. Un freak enterre un freak [...] la Vie en prose devrait finir comme ça, répète Julien à trois reprises (p. 243). Dans les dernières pages du chapitre 9, Nane enterre sa liaison freakante avec Raël qui la téléguidait; elle décide d'en finir avec cette relation basée sur la peur, sur la honte, sur l'horreur. On sait aussi que la Vie en prose a été écrite entre 1976 et $1979^{22}$. Le neuvième chapitre se clôt sur un bilan: Trois ans, jour pour jour, depuis ce jour où j'ai écrit la première phrase de cette vie en prose... ((p. 223). Comme s'il restait des doutes à dissiper: Une fois le dernier mot écrit et C'est pour lui que j'ai écrit, ajoute-telle (p. 220 et 223). 


\section{Maintenant le Livre-Sphynx}

Comme la Vie en prose, il a une structure tripartite. Il débute par «le livre-sphynx», lieu du commencement, de l'écriture qui s'ébranle, des événements de l'été 78 (assassinat d'Aldo Moro) en A, puis passe par Yvelle Swannson en $\mathrm{B}$, qui regarde vers le temps de l'avant (p. 201) pour

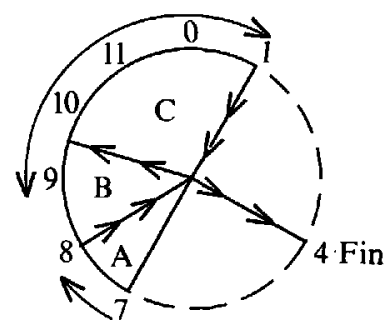

Début * finir par "l'écriture rouge» (C) dont les différents épisodes couvrent les mois de juin et juillet de l'année 79 (préparation du référendum, «ça va être les années $80 »-$ p. $255-$, le skylab). La toute fin cependant, le "happy-end" depuis longtemps annoncé se produira au chapitre quatre de la Vie en prose (cette fois, c'est le Yang dans le Yin) qui

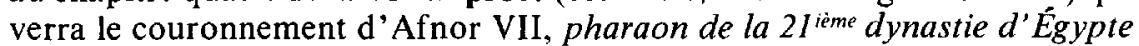
(p. 82). Conclusion toute désignée pour un livre-sphynx. Fiction logique. Logique de la fiction.

La lecture circulaire compose une nouvelle diégèse. Une seule et même narratrice ( $Y$.) écrit en même temps deux romans sous des noms différents. Dans chacun de ces romans, une narratrice se dédouble également pour donner vie à d'autres narratrices qui se dédoubleront à leur tour. Y. a parfois de la difficulté à circuler dans le labyrinthe qu'elle a elle-même conçu. C'est ce qui arrive quand on joue tous les rôles et qu'on les double en plus (p. 171). Un tableau synoptique permet de mieux visualiser le phénomène de duplication systématique des personnages (avec une marge d'erreurs croissante vers la dernière colonne car plus il y a de substitutions, moins les héroïnes sont identifiables).

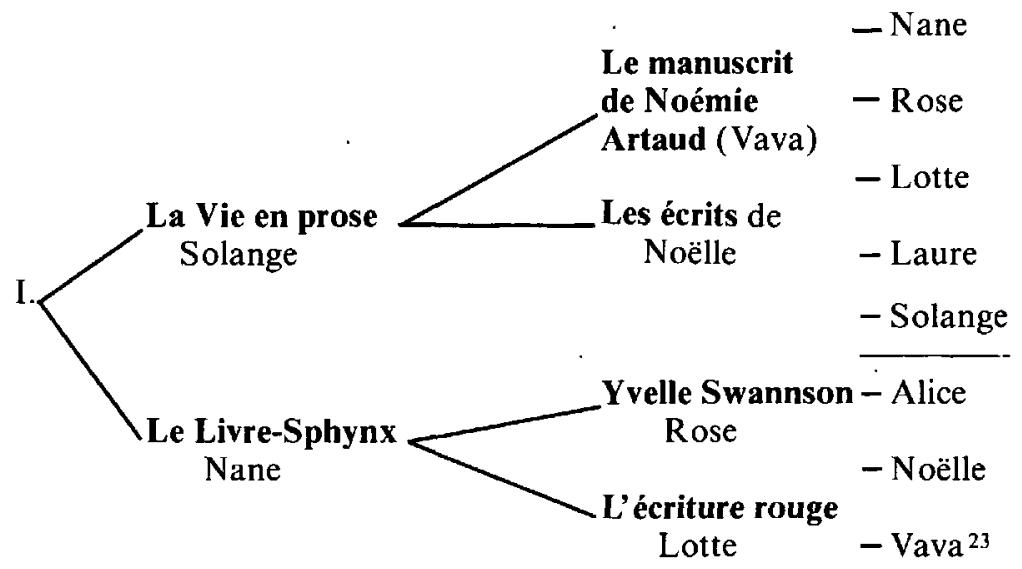


L'architecture du roman autorise plus d'un parcours textuel. Rien n'empêche de considérer l'ouvre comme une étroite imbrication de deux textes dont les feuillets s'entremêlent ( $S a$ version s'enchâsse dans la mienne comme la mienne dans la sienne p. 223), bousculant la chronologie, dans un univers où il est question de deux géographies à la fois (p. 207). Lecture doublement circulaire qui saute des morceaux de temps et d'espace et qui oblige le lecteur à repass [ er ] sur toutes [ $l$ ] es traces (p. 42) pour recomposer la trame événementielle, pour reprendre le fil du récit.

Mais dans tous les cas, la Vie en prose se déploie et se déroule autour de la question de l'identité. Dans le dédale onomastique s'engrènent les effets spéciaux. Au premier chapitre, N.Y. (Nane Yelle) arpente L.A. (Los Angeles) et dans le deuxième, L.A. (Lotte Arbour) se trouve à N.Y. (New-York). L'échangeur maniaque des destins (p. 204) fabrique des capitales de noms propres: N.Y. c'est aussi Noëlle Yelle; L.A. c'est aussi Laure de son nom d'Aurel. Parfois il condense: N.A. (Noémie Artaud); parfois il réfléchit: Alice se mire en Celia.

Or la suractivité du texte ne doit pas nous faire perdre les lignes directrices. Il y a d'une part le déguisement du je qui entre dans la machine fictive pour se perdre aussitôt dans le regard de ses sosies, toutes chevelures liées (p. 216). Le je qui se dit dans ce roman-gigogne en expansion vers son point de fuite (p. 250) ne peut, dans un premier temps, que s'égarer dans le labyrinthe infernal de ces braves et bonnes héroïnes enchâssées jusqu'au vertige (p. 185).

D'autre part, ce je (qui est ici un Y) s'inscrit comme en «réimpression sur la fiction". Au centre du livre, un tout petit livre, le livre-sphynx qui est signé d'un $Y$ et d'un $V$ renversé (comme sur le tableau de la page 16): initiales identiques à celles de l'auteure, dont le nom apparait sur la couverture. De ce nom (Yolande Villemaire) qui se tient hors du jeu pour le circonscrire, le prénom se détache et entre dans le jeu machinal pour s'y donner à lire d'abord voilé (Une voix proclame que... p. 8), puis sous la forme d'un surnom ( (Yoyo", p. 207) et enfin, en entier mais subrepticement ( Yolande») à la dernière page du livre. Nom et prénom apparaîtront ensemble sous une forme anagrammatique ( Noé-Vladimira Yelle» p. 79).

Il y a émission (mise en circulation) du je dans la fiction, ce je qui est un même, qui est un autre, qui est pluriel. Le jeu du je en est un de substitution, de déformation, de multiplication. Une présence qui s'absente... en se multipliant. Passe encore Aurélia suivie de toutes les filles du feu.

\section{Strass, cristal et quartz}

A circuler dans le dédale en forme de livre, on se rend compte qu'en plus d'être fixés dans la prose, les indices sont parfois inscrits dans la pierre. Le motif lapidaire varie au gré des pages; du rude au chatoyant, il prend des aspects divers. Au centre du livre, un manuscrit: le Livre-Sphynx. A l'intérieur du manuscrit, un peu excentrique, une pierre: le livre-sphynx dont on nous dit approximativement les dimensions et la forme: C'était un très petit 
livre-sphynx, la pierre ne devait pas avoir plus d'un pied de haut et un pied de large, mais elle n'était pas carrée (p. 149). La promeneuse y appose ses initiales entrelacées dans la magie du chiffre (p. 145) ${ }^{24}$. C'est la pierre angulaire du récit (bien qu'elle soit à peu près au centre) ${ }^{25}$, la matière brute, le trou noir qui aspire et résume ce qui gravite autour de lui.

Le roman n'utilise pas que de tels matériaux. Souvent les pierres sont cristallines, complices du jeu de la lumière; elles s'apparentent au verre, possèdent des pouvoirs magiques. Le «métallique" participe de cette constellation. Les vaisseaux spatiaux s'illuminent et les trains roulent $[\ldots]$ dans de grands flashes de kryptonite (p. 194). Mais la pierre-signe brille parfois d'un éclat trompeur; l'indice est en même temps piège: comment identifier tous ces danseurs qui virevoltent sur la piste dans des éclats de strass, de paillettes, de quartz?

A l'état pur, le quartz est cristal. Le cristal renvoie aux miroirs qui pullulent dans le roman. Trois fois passera... par le Crystal Palace Tango ${ }^{26} \mathrm{le}$ lecteur d'Yvelle Swannson qui regarde à travers le cercle de plexiglass ( $\mathrm{p}$. 193) ou par la vitre parabolique (p. 194) le soleil qui plombe sur la mer de Moscou. À moins qu'il ne voit plutôt le paysage dans l'une des nombreuses boules de cristal que le roman distribue à la ronde. Indice, signe, oui. Mais que faire des cristaux de lumière éclatée de la boule en miroir? (p. 199).

Il faut chercher le diamant. Or il a un indice de réfraction élevé et un égal pouvoir de dispersion. En tout cas, il est magique. Il garantit le passage de l' "autre côté"; le diamant d'Amsterdam assure le transfert de ce monde dans un autre monde (p. 82). Lorsqu'elle appuie sur le losange de cristal fixé à son front, Yvelle Swannson se pulvéris [e] dans l'hyperespace (p. 183).

On tombera finalement sur la pierre la mieux cachée, la plus estimable, l'escarboucle mystérieuse (p. 98) que le Petit Robert définit ainsi: Pièce héraldique qui représente une pierre précieuse d'où partent huit rais terminés par des fleurs de lis. Elle est de la même lignée indiciaire que le livre-sphynx, autre pierre précieuse autour de laquelle rayonnent huit personnages échappés d'un même centre.

\section{L'important, c'est la rose...}

Curieux et remuants, les personnages de la Vie en prose cherchent leur Passage to India. Ils s'initient au bouddhisme tantrique, s'adonnent au yoga kundalini, s'intéressent à la spiritualité tibétaine. On reconnaît la ferveur des néophytes à leur vocabulaire chargé de kas, de karmas, de shakras, de mudras, de mandalas, etc., sorte d'India Song remodulé pour la circonstance. Sans vouloir exploiter à fond tous les détours de ce nouvel itinéraire, il vaut la peine de montrer comment il se surajoute au cycle précédemment décrit.

Une des narratrices s'aventure dans des cryptes à la recherche de vieux missels oubliés et du premier cercle vicieux samsarique (p. 202). Or, le samsara est un terme sanscrit qui indique: 
le cycle des naissances et des morts, [...] que symbolise dans l'art hindou une roue à six, huit ou douze rayons autour du moyeu [...] Le moyeu figure le centre où doit revenir la conscience pour y découvrir la paix [.... Faute de quoi nul n'échappera à la roue des existences, dans une suite indéfinie de réincarnations ${ }^{27}$.

Parenté évidente avec la structure circulaire du roman qui reproduit son héroïne jusqu'au vertige.

A la fin du roman, Nane raconte qu'elle s'est fait tirer aux pyramides. Elle a posé une question: la pyramide a répondu: It will blossom like a flower (p. 256). Dans les représentations de la philosophie orientale, le lotus qui épanouit ses six pétales autour d'un centre rond, dessine une figure que l'on retrouve dans le roman: six manuscrits gravitent autour du livre-sphynx.

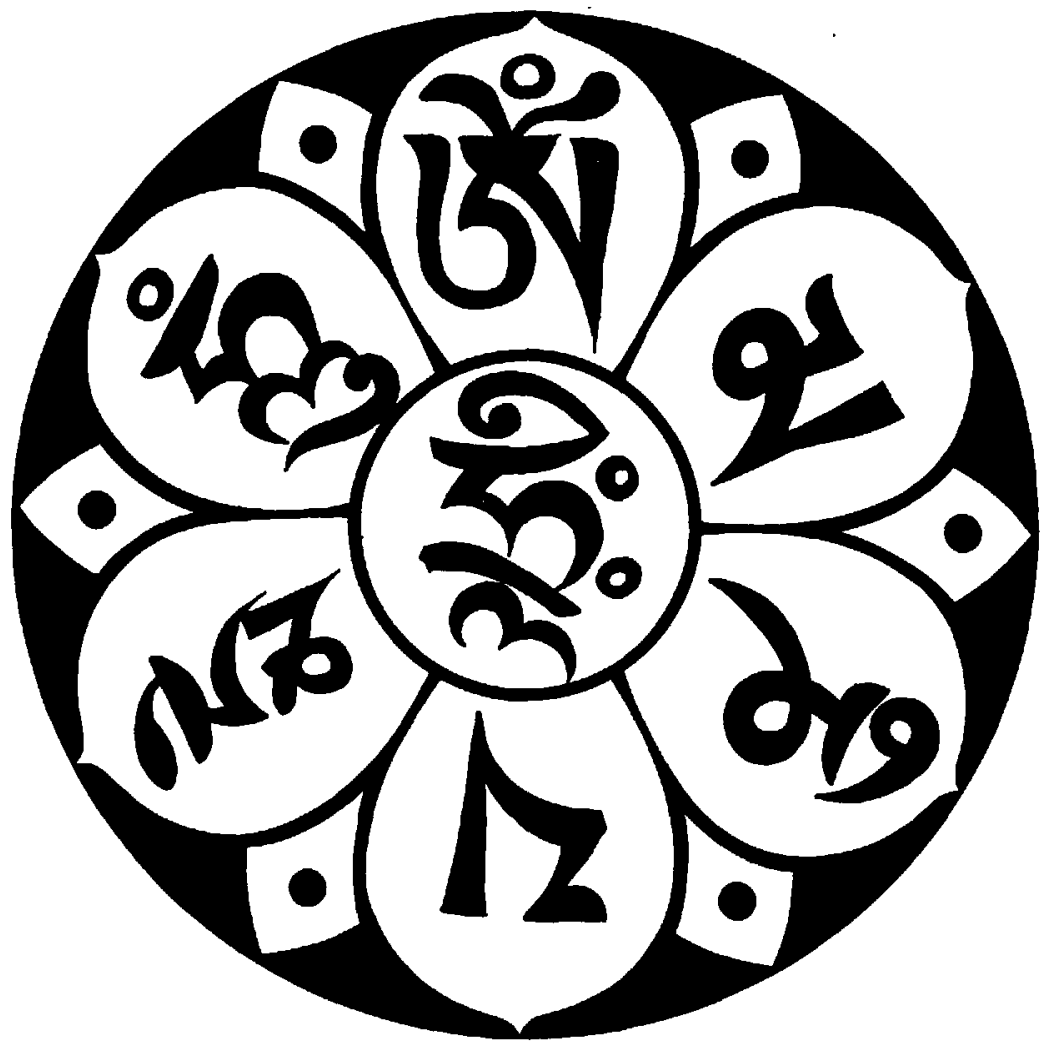

Par ailleurs, le lotus est souvent associé à la rose dont on dit qu'elle est $l a$ fleur symbolique la plus employée en Occident. Elle correspond $[\ldots]$ à ce qu'est le lotus en Asie, l'un et l'autre étant très proches du symbole de la roue ${ }^{28}$. 
A plusieurs reprises, les personnages disent avoir entendu "le son" primordial hindou. On ne peut le lire entier, toutefois, que vers la fin: Om-Mane-Padme-Hum (p. 215). Il est essentiel dans la spiritualité tibétaine, ajoute le Dictionnaire des symboles, et il est représenté par «le joyau dans le lotus". Qu'on se rappelle l'escarboucle du chapitre précédent et que l'on garde à l'esprit en "contemplant" la figure ci-contre le contexte qui entoure la signature du livre-sphynx: J'avais écrit un caractère tibétain sur ceite pierre qui avait l'air d'un livre [...] Et c'était mon nom que j'écrivais car ce caractère. graphiquement $[\ldots]$ en représentait les initiales entrelacées dans la magie du chiffre trois ( $\mathrm{p}$. 148).

\section{Précis d'optique}

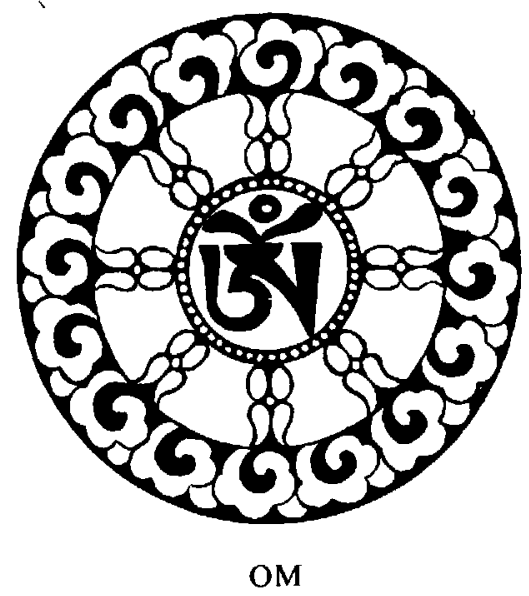

THE PATH OF UNIVERSALITY 29

Dans un article récent, Villemaire écrit: Le futur n'est qu'une image en train de se former sur notre écran mental [...] Chaque pensée, chaque mot, chaque geste font une empreinte dans le cosmos et dessinent l'hologramme $d u$ futur ${ }^{30}$. Sa fascination pour l'hologramme transparaît dans la Vie en prose où les personnages recourent à son principe pour articuler le comment des choses (p. 254).

Insolite, la structure du roman rappelle, elle aussi, le monde à la fois réel et irréel de l'holographie. Grâce à la technique photographique sophistiquée de l'imagerie tridimensionnelle, les objets «enregistrés» peuvent maintenant être reproduits et visualis[és] avec leur relief intégral ${ }^{31}$. Le plan fixe de la photographie est dépassé. L'image obtenue constitue un nouvel objet: on peut en faire le tour, son aspect change avec la position de l'observateur. Toutes considérations qui ne sont pas étrangères aux desseins (dessins) du roman.

La fabrication de l'hologramme exige un laser? La Vie en prose en arbore (p. 192, 195, 199). Cette source lumineuse monochromatique émet un rayonnement qui est divisé en deux faisceaux au moyen d'une lame séparatrice $^{32}$ (au chapitre 7 le livre est littéralement séparé en deux parties), l'un éclaire grâce d̀ une optique appropriée l'objet à holographier, l'autre est renvoyé par l'intermédiaire d'un miroir sur la plaque photographique ${ }^{33}$ (qu'on se souvienne de l'effet de miroir extrêmement précis du chapitre 4).

Une fois enregistrés, ces deux procédés ne donnent pas immédiatement accès à l'hologramme. Le développement de la plaque montre un brouillage d'ondes, une juxtaposition d'interférences. Il en va de même du roman. La première lecture ne fait voir que superpositions de personnages, qu'entrecroi- 
sements de lignes. Une autre opération (décodage, restitution) livre l'objet final.

Question: la Vie en prose chercherait-elle à établir de nouveaux ponts entre la science et la fiction?

\section{Le titre, à titre illustratif}

Il y a parfois inadéquation du titre et du texte, note Jean Ricardou. Un titre peut promettre davantage qu'il ne tient ou tenir davantage qu'il promet ${ }^{34}$. Peu importe qu'il soit en hyperbole ou en litote, le titre de cet essai, par la figure de l'ourobouros, insiste plutôt sur l'aspect circulaire et cyclique du roman. Dans la structure ronde des deux livres enchâssés, les mêmes événements se reproduisent, présentés par différentes visions narratives. Cette version, le roman la contient en abyme:

C'est à se demander si ça n'a pas quelque rapport avec cette publicité du métro de Paris sur laquelle on voit une série de filles brunes en bikini $[\ldots]$ d'une station à l'autre [...] Le jour où l'on s'étonne que toutes les Françaises soient aussi brunes, on découvre que c'est toujours la même fille, cheveux dénoués ou mouillés, avec lunettes-soleil ou sans.

Le retour du même (bien que travesti) y est éminemment présent. Mais ce dont le serpent mythique ne rend pas suffisamment compte c'est du mouvement hélicoïdal qui anime le roman.

Qu'il s'agisse du cahier spirale (p. 14), de la spirale de nuages (p. 160), de la spirale vénitienne ( $\mathrm{p}$. 161) ou de la spirale du temps (p 171), le mot revient souvent, diversement assorti. Le texte insiste sur le mouvement: Ça se met à tourner très vite [...] ça tourne, ça tourne, une force prodigieuse m'aplatit contre la paroi [...] et ça tourne, et ça tourne (p. 246). Les personnages tourne [ $n t]$ dans des arcs-en-ciel (p. 247) et prennent plaisir à jouer les derviches-tourneurs (p. 99, 162, 181, 196, 202).

Pour Chevalier et Gheerbrant, la spirale est un motif ouvert et optimiste (la vie en rose); elle manifeste l'apparition du mouvement circulaire sortant du point originel; ce mouvement, elle l'entretient et le prolonge à l'infini ${ }^{35}$, rejoignant ici la symbolique du huit déjà entrevue. Les représentations spiralées évoquent le labyrinthe, se rattachent au symbolisme vulvaire. La double spirale enroule les serpents autour de la caducée; elle représente l'équilibre dans le déséquilibre, l'identité de l'être ạu sein du changement.

Ourobouros? Kundalini? Peu importe la figure qu'il emprunte, le serpent, ligne vivante, sans commencement ni fin, susceptible de toutes les représentations et de toutes les métamorphoses, semble on ne peut plus apte à dire l'univers mouvant et rusé de ce roman reptiligne. 


\section{The game is over}

Fiction. Le lieu de ralliement de la modernité. Mais la Vie en prose avec son jeu d'accessoires et de déguisements estompe et entremêle à plaisir les lignes du réel et les voies du rêve, les traits de la vie et de la graphie, le fil des mots, le grain des choses. Jusqu'où traquer le réel quand le réalisme est possible en littérature mais que la littérature n'est pas possible en réalité? Jusqu'où remonter la fiction quand on touche les cordons ombilicaux des hérö̈nes aux cœurs qui battent comme des bolos? Roman qui contient le principe de sa prolifération, qui machine une série de divertissements, de diversions, de substitutions, qui programme et déguise le retour du même à tel point qu'on ne parvient jamais à toucher «l'origine», «le premier terme», "l'authentique sujet», ce qui se tient hors l'écrit mais qui s'y livre en même temps témoignant par là de l'amour ex aequo de la vie et de la prose, jouant à déplacer la ligne de frontière entre la réalité et la fiction, produisant là ce «tremblement du réel» qui est peut-être l'enjeu de la modernité.

Pour l'heure, refermons le jeu en forme de livre. The game is over. Jusqu'au prochain lecteur. Le nouveau mode d'emploi.

1. Yolande Villemaire, la Vie en prose, Montréal, Les Herbes rouges, 1980, p. 128. Dorénavant, les renvois à cet ouvrage seront inclus dans le texte, entre parenthèses.

2. Jean Ricardou. "La population des miroirs. Problèmes de la similitude à partir d'un texte d'Alain Robbe-Grillet", Poétique 22, 1975, p. 202.

3. Ibid., p. 201.

4. La Vie en rose qui naîtra du Temps fou en mars 1980, quelques mois avant la publication du roman mais après sa rédaction.

5. René Bertelé, préface de 1968 à Corps et biens de Robert Desnos, Paris, Gallimard, 1968, p. 7.

6. Yolande Villemaire, «Holding pattern», Vouloir la fiction $\odot$ la modernité, Montréal, Éditions de la nbj, 1984, p. 71.

7. Raymond Montpetit, Comment parler de la littérature, Montréal, Cahiers du Québec / Hurtubise HMH Ltée, 1976. p. 125. (Coll. «Philosophie»).

8. Ibid., p. 11 .

9. Ibid., p. 123 .

10. Ibid., p. 12.

11. Ibid., p. 125.

12. Réginald Martel, «Pourquoi écrire aujourd'hui? Un secret bien gardé», La Presse, Montréal, samedi 13 octobre 1984, p. E 3. Ibid pour la citation suivante.

13. Jacques Derrida. "La pharmacie de Platon», la Dissémination, Paris, Seuil, 1972, p. 71.

14. Ibid., p. 71 .

15. Nous soulignons les deux substantifs car ils rendent compte du lien entre la figure du huit et l'aspect non seulement thématique mais aussi structural du roman (p. 151).

16. A la fin du chapitre $V$, p. 101 à 106.

17. C'est nous qui soulignons, (p. 127).

18. C'est nous qui soulignons, (p. 81). 
19. Cette interprétation se justifie par un faisceau d'informations recueillies en page 80 . Le manuscrit couvrirait «le quart de la surface» du roman. Or la Vie en prose de Solange contient 48 scènes et le chapitre I en comprend 12, soit précisément le quart.

20. L'expression "le quart de la surface» s'applique-t-elle au roman de Solange ou à la Vie en prose de Y. Villemaire. Dans ce dernier cas, le livre comprend 100 scènes: la surface est doublée.

21. L'allusion à la doctrine chinoise n'est pas gratuite. Le mot Tao veut dire chemin, voie, et le roman y a recours à plus d'une reprise. On y est fasciné par les dix mille choses du Tao Te King dont l'infinie variété n'est ni réelle ni illusoire mais les deux à la fois (p. 126).

22. Voir la note en italique à la page 262 .

23. C'est le chiffre 26, rappelons-le, qui préside à cette structure. On le rencontre sous forme de dates: 26 décembre (p. 126), 26 mars (p. 219); il désigne l’âge de deux personnages, 26 ans (p. 151); on nous dit que le deux binaire du duel et le six démoniaque et pluriel ont servi de techniques d'enchâssement (p. 210) et que 26, en calcul théosophique, ça donne huit (p. 15I). Voilà: deux livres principaux se divisent en six manuscrits qui racontent les aventures de huit personnages-écrivains. Pourtant, cette lecture n'épuise pas les possibilités formelles du roman. Un certain nombre de pistes (qui n’ont pas été étudiées en détail) semblent mener à une structure quadripartite comme celle qui est suggérée par l'arcane XXI du Tarot de Marseille (autre chemin initiatique) dont la figure circulaire est entourée de quatre symboles: l'ange. l'aigle, le bœuf, le lion ( p. 125). Cette nouvelle partition pourrait s'ordonner autour de la roue des saisons, fortement marquées dans le texte, et en regard des quatre éléments ("épisode du feu», "épisode de la neige»). Grosso modo, elle pourrait ressembler à ceci:

$\begin{array}{llll}\text { TERRE } & \text { AUTOMNE } & \text { LIRE } 1+2+8=25 \text { scènes } & \text { BCEUF } \\ \text { AIR } & \text { PRINTEMPS } & \text { LIRE } 3+4+11=25 \text { scènes } & \text { AIGLE } \\ \text { EAU } & \text { HIVER } & \text { LIRE } 5+6+9=25 \text { scènes } & \text { ANGE } \\ \text { FEU } & \text { ÉTÉ } & \text { LIRE } 7+10=\frac{25 \text { scènes }}{100 \text { scènes }} & \text { LION }\end{array}$

Des correspondances textuelles relient les chapitres groupés. Par exemple, la comparaison comme dans l'électrocardiogramme d'un mourant dont le cour s'emballe lue au chapitre 1 se réverbère mot à mot dans le chapitre 8 (p. 15 et 174).

24. Ce n'est pas la première fois que la pierre est associée au chiffre trois; un des personnages masculins s'appelle Pierre-Pierre-Pierre.

25. Il s'agit d'un roman de 262 pages. L'épisode du livre-sphynx se situe à la page 148 .

26. p. 195, 197, 198.

27. Jean Chevalier et Alain Gheerbrant, Dictionnaire des symboles, Paris, Robert Laffont/Jupiter, 1969/1982, p. 842.

28. Jean Chevalier et Alain Gheerbrant, Dictionnaire des symboles, p. 822.

29. La figure est tirée de Foundations of Tibetan Mysticism, p. 15.

30. Yolande Villemaire, «Dessiner l'hologramme du futur», Le Devoir, Montréal, samedi 17 nov. 1984, Cahier I, p. 1 et 26.

31. Jean Bulabois et Gilbert Tribillon, «Les images à trois dimensions», la Recherche. La révolution des images, p. 638 .

32. Ibid., p. 640.

33. Ibid., p. 640.

34. Jean Ricardou, “La population des miroirs", p. 202.

35. Jean Chevalier et Alain Gheerbrant, Dictionnaire des symboles, p. 906. 


\section{BIBLIOGRAPHIE}

BULABOIS, Jean et Gilbert Tribillon, "Les images à trois dimensions», La Recherche. La révolution des images, no 144, mai 1983, p. 638-649.

CHEVALIER, Jean et Alain Gheerbrant, Dictionnaire des symboles, Paris, Robert Laffont/ Jupiter, $1969 / 1982$.

DER RIDA, Jacques, "La pharmacie de Platon ". la Dissémination, Paris, Seuil, 1972, p. $71-95$.

DESNOS, Robert, Corps et biens. Paris. Gallimard. 1968, 190 p. (Collection «Poésie»).

GOVINDA, Anagarika, Foundations of Tibetan Mysticism, London, Rider \& Company, 1969, $311 \mathrm{p}$.

HAECK, Philippe, la Table d'écriture. Poéthique et modernité, Montréal, VLB Éditeur, 1984, $386 \mathrm{p}$.

MARTEL, Réginald, “Pourquoi écrire aujourd'hui? Un secret bien gardé. La réponse des écrivains", La Presse, Montréal, samedi 13 octobre 1984, p. E-3.

MONTPETIT, Raymond, Comment parler de la littérature, Montréal, Cahiers du Québec /Hurtubise HMH Ltée, 1976, 195 p. (Collection "Philosophie").

RICARDOU, Jean, «La population des miroirs. Problèmes de la similitude à partir d'un texte d'Alain Robbe-Grillet". Poétique 22, 1975.

VILLEMAIRE, Yolande, "Dessiner l'hologramme du futur", Le Devoir. Montréal, samedi 17 novembre 1984, Cahier I. p. 1 et 26.

VILLEMAIRE, Yolande, «Holding pattern». Vouloir la fiction $\odot$ la modernité. Montréal. Éditions de la nbj, 1984, p. 71-72.

VILLEMAIRE, Yolande, la Vie en prose, Montréal, Les Herbes rouges, 1980, 262 p. (Collection "Lecture en vélocipède"). 